Results Four critically ill children (aged 1-14 years), requiring resection of ischaemic colon following sudden cardiovascular collapse, presented to our institutions over a 2-year period.

Three had a preceding history of recent illness; the other had been well prior to out of hospital cardiac arrest. The 3 who were unwell experienced: headache (1), cough (1) and polyuria and polydipsia (1) for up to 2 weeks prior to hospital attendance. None had abdominal or gastrointestinal (GI) symptoms in their initial symptoms, although all but 1 developed GI upset during their rapid deterioration phase. Three had cardiac arrest before colectomy. All developed abdominal distension after resuscitation. All had significant derangement of blood sugar on monitoring. All 4 received inotropic support before surgery. All 4 had total colonic ischaemia diagnosed during surgical intervention and on histology report. There was no other underlying disease on histology (bowel was ganglionic). No infective organism was isolated (specifically all were negative for clostridium difficile). All 4 died due to multi organ failure.

Summary and Conclusion From this case series we could not identify any specific condition that predisposed these 4 children to develop non occlusive mesenteric ischaemia and colonic injury. Therefore we could not clearly identify means of prevention.

All 4 had a sudden deterioration over less than 24 hrs, and all received inotropes before developing abdominal distension. In addition, 3 out of 4 had cardiac arrest and return of circulation before colectomy. These would support the hypothesis of hypoperfusion/reperfusion injury.

Even though all 4 patients had colonic resection as part of the resuscitation the outcomes were very poor leading to multi organ failure or cerebral ischaemia and death.

\section{P49 SHOULD CHILDREN WITH COELIAC DISEASE BE SCREENED FOR TYPE 1 DIABETES MELLITUS IN ANNUAL BLOODS? AN AUDIT OF FOUR YEARS SCREENING ACROSS FOUR PAEDIATRIC CENTRES IN ENGLAND}

${ }^{1}$ Arati Rao, ${ }^{2}$ Siba Paul, ${ }^{3}$ Ozan Hanci, ${ }^{1}$ Cynthia Diaba, ${ }^{4}$ Mai Abdalla, ${ }^{2}$ Winnette Akpobire, ${ }^{4}$ Shiv Tibrewal, ${ }^{1}$ Mark Furman. ${ }^{1}$ The Royal Free Hospital; ${ }^{2}$ Torbay Hospital; ${ }^{3}$ Royal Surrey County Hospital; ${ }^{4}$ Darlington Memorial Hospital

\subsection{6/flgastro-2021-bspghan.58}

Introduction The genetic association of Coeliac Disease (CD) and Type 1 Diabetes Mellitus (T1DM) is well known. Although NICE does not include annual screening for CD in the T1DM guidance, ${ }^{1}$ children with T1DM are routinely screened for CD in England. The incidence of children with CD on gluten free diet (GFD) developing T1DM appears to be small. ${ }^{2}$ The value of screening for T1DM in CD patients is thus not known.

Methods A 4-year retrospective review was conducted of a case series of children with known $\mathrm{CD}$ attending outpatient clinic across 4 centres. Patients were diagnosed as per ESPGHAN guidelines. In these centres HbA1c is tested as part of the $\mathrm{CD}$ annual review to detect impairment of glucose metabolism (> $41 \mathrm{mmol} / \mathrm{mol}$ ). Abnormal HbA1c was documented in patients with CD.

Results 345 children with CD who had HbA1c screening were identified. Children with T1DM diagnosed prior to developing CD were excluded from analysis. Six of the 345 patients
(1.7\%) were identified with an abnormal HbA1c. Only 2/6 were confirmed as having T1DM (1 within 4 months of diagnosis); $2 / 6$ had subsequent glucose tolerance tests that were normal, 1 patient had Turner's syndrome and was taking growth hormone, which has an impact on glucose metabolism, and 1 patient is undergoing further investigations for Type 2 Diabetes Mellitus (high BMI).

Discussion The findings confirm the conclusion of previous studies that showed that a new diagnosis of T1DM in known CD children is uncommon. The annual screening for T1DM in children with $\mathrm{CD}$ who have developed impaired glucose tolerance is questionable. There is no standard screening test for T1DM, and HbA1c as a screening test for T1DM is also not routinely used. The exact mechanism for expressing coexisting autoantigens that generate both autoimmune conditions is poorly understood, and it is not clear whether the GFD in CD plays a role.

\section{REFERENCE}

1. National Institute of Health and Care Excellence (NICE) (2020) 'Management of children and young people with type 1 diabetes'. Available at: https://cks.nice. org.uk/topics/diabetes-type-1/management/management-children-young-people/

2. Ludvigsson JF, et al. 'Celiac disease and the risk of subsequent type 1 diabetes'. Diabetes Care 29:2483-2488, 2006

\section{P50 SINGLE CENTRE EXPERIENCE OF ENDOSCOPIC BALLOON DILATATION FOR LUMINAL STRICTURES IN PAEDIATRIC PATIENTS WITH CROHN'S DISEASE}

Kirn Sandhu, Vinod Kolimarala, Babu Vadamalayan. King's College Hospital

\subsection{6/flgastro-2021-bspghan.59}

Introduction/Background Intestinal strictures are a complication of Crohn's disease despite optimal medical management. Endoscopic balloon dilatation is frequently used for management of simple strictures in adults in comparison to the paediatric population. Therapeutic endoscopy is rarely performed in paediatric gastroenterology centres in the UK. Strictures in the duodenum, jejunum, ileal and colonic area are accessible by endoscopy and enteroscopy. Endoscopic balloon dilatation is a less invasive treatment option for management of short strictures and can defer surgical intervention.

Aim We aimed to evaluate the outcome of paediatric patients undergoing stricture dilatation over a 10 -year period.

Subjects and Methods We retrospectively reviewed all paediatric patients with Crohn's disease who underwent endoscopic balloon dilatation at a tertiary paediatric gastroenterology centre in the last 10 years (2010 to 2020). Strictures were identified using magnetic resonance enterography (MRE) and also during endoscopy. Patients were booked for endoscopic balloon dilatation if they were symptomatic and had pre-stenotic dilatation on MRE or inability to pass colonoscopy into stenosed lumen at previous endoscopy. Both paediatric colonoscopy and single balloon enteroscopy was used for endoscopic balloon dilatation which was done under fluoroscopy guidance. Clinical and endoscopic data were collected from electronic patient records.

Results During the 10-year period 20 patients with Crohn's disease underwent endoscopic balloon dilatation. The mean age of diagnosis of Crohn's disease was 12.45 years (5$16.4 \mathrm{yrs})$ and the mean age at the time of the occurrence of first stricture was 14.2 years (10.9- 17.9 yrs). $65 \%$ patients were on biologics and $85 \%$ were on an 